\title{
Prediction of white matter integrity pattern in overweight and obese adults
}

\author{
Sussanne Reyes ${ }^{1}$, Patricio Peirano ${ }^{1}$, Betsy Lozoff ${ }^{2}$ and Cecilia Algarin ${ }^{1}$ \\ ${ }^{1}$ Laboratory of Sleep and Functional Neurobiology, Institute of Nutrititon and Food Technology (INTA), University of \\ Chile, Santiago, Chile and \\ ${ }^{2}$ Professor Emerita, Department of Pediatrics and Communicable Diseases, University of Michigan, Ann Arbor, USA
}

\section{Abstract}

Introduction

Obesity has been associated with lower white matter integrity (WMI) in limbic brain regions, including the fornix. Both early decrease of WMI in the fornix (WMIf) and midlife obesity have been related to dementia incidence with advancing age. No studies have explored early cognitive predictors of WMIf in overweight-obese $(\mathrm{OO})$ adults. Aim of this study was to compare OO and normalweight (NW) participants with respect to (a) WMIf in adulthood and (b) the relationship between cognitive performance at school-age and in adolescence with WMIf in adulthood.

\section{Methods}

Participants were part of a cohort followed since infancy who underwent magnetic resonance imaging studies in adulthood (22.3 \pm 1.3 years). Diffusion tensor imaging was performed and Tract Based Spatial Statistics (TBSS) was used to obtain fractional anisotropy (FA) skeleton; increased FA relates to greater WMI. A mask for the fornix was created (JHU-ICBM DTI-81 Atlas) and then used to extract the average FA for each individual. Participants also performed neurocognitive tasks: (a) school-age (10.3 \pm 1.0 years): the trail making test comprises two conditions and time difference between conditions reflects cognitive flexibility; (b) adolescence (15.6 \pm 0.5 years): incentive task that test the effect of incentives (reward, loss avoidance or neutral) on inhibitory control performance (correct responses latency). In adulthood, BMI was categorized as NW ( $\geq 18.5$ to $<25.0 \mathrm{~kg} / \mathrm{m} 2)$ and OO ( $\geq 25.0 \mathrm{~kg} / \mathrm{m} 2)$ groups. A t-test and univariate GLM were conducted. Analysis were adjusted by sex and age-specific BMI z-scores.

\section{Results}

Participants were $27 \mathrm{NW}$ (41\% female) and 41 OO (49\% female). Compared to NW, OO participants showed decreased FA in the fornix $(0.585$ vs. $0.618, \mathrm{p}<0.05)$, i.e. lower WMIf. Differences were apparent in the relationship between cognitive flexibility at school-age $(F=2.9, p=0.06)$ and loss avoidance latency in adolescence $(F=3.5, p<0.05)$ with FA in the fornix in adulthood. Increased cognitive flexibility at school-age $(\beta=0.335, \mathrm{p}<0.05)$ and decreased loss avoidance latency in adolescence $(\beta=-0.581$, $\mathrm{p}<0.001$ ) were related to higher FA in the fornix in OO adults. No relationship resulted significant in NW adults.

\section{Discussion}

Performance in neurocognitive tasks at earlier developmental stages were related with WMIf only in OO adults, group characterized by decreased WMIf. Our results provide evidence regarding specific neurocognitive tasks with predictive value for WMIf alterations. Further, they could contribute to the understanding of neural mechanisms underlying obesity and also provide insight relative to neurodegenerative risk with advancing age.

\section{Support}

Fondecyt 11160671 and NIH HD33487.

\section{Conflict of Interest}

There is no conflict of interest 